\title{
The Investigation of the Magnetization Reversal Mechanism in the Nd-Fe-B Type Magnet, Aligned by Hot Deformation
}

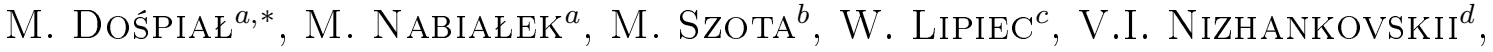 \\ A.E. CeglareK ${ }^{a}$ And P. PietrusiewiCz ${ }^{a}$ \\ ${ }^{a}$ Institute of Physics, Częstochowa University of Technology, al. Armii Krajowej 19, 42-200 Częstochowa, Poland \\ ${ }^{b}$ Institute of Materials Science, Częstochowa University of Technology \\ al. Armii Krajowej 19, 42-200 Częstochowa, Poland \\ ${ }^{c}$ Electrotechnical Institute, M. Skłodowskiej-Curie 55/61, 50-369 Wrocław, Poland \\ ${ }^{d}$ International Laboratory of High Magnetic Fields and Low Temperatures, Gajowicka 95, 53-421 Wrocław, Poland

\begin{abstract}
The magnetization reversal mechanism in the $\mathrm{Nd}_{16} \mathrm{Fe}_{78} \mathrm{~B}_{6}$ hot densified magnet, aligned by means of die-upset forging has been investigated. The magnetic parameters have been derived from major hysteresis loop. The magnetocrystalline anisotropy constants $K_{1}$ and $K_{2}$ using Sucksmith-Thompson relation modified by Ram and Gaunt have been calculated from the high field measurements up to $5 \mathrm{~T}$. These data have been used to determine the theoretical value of coercivity as a function of the angle $\Psi_{0}$ between the sample easy axis and the applied magnetic field direction. The experimental value of coercivity as a function of $\Psi_{0}$ has been determined from the demagnetization curves measured for different $\Psi_{0}$ angles. It was found that the best correlation between theoretical and experimental data have been achieved for magnetization reversal mechanism controlled partially by pinning of domain walls on grain boundaries and nucleation processes.
\end{abstract}

PACS: $75.30 . \mathrm{Gw}, 75.30 . \mathrm{Kz}, 75.50 \mathrm{Vv}, 75.50 . \mathrm{Ww}, 75.60 . \mathrm{Jk}$

\section{Introduction}

Anisotropic magnets prepared on the basis of $\mathrm{Nd}-\mathrm{Fe}-\mathrm{B}$ compound, by hot pressing of hard magnetic powder $(M Q 2)$ and then aligned in the hot deformation process (MQ3) belong to a group of hard magnetic materials, with excellent magnetic properties. Their unique properties are determined by: high density of the final magnet, and a significant degree of crystallographic texture. The densities of the produced magnets are in range from $91 \%$ to $99.6 \%$ of the densities of powders used in production process [1]. The crystallographic texture, obtained in the deformation process, results in formation of large magnetocrystalline anisotropy, which has significant influence on the magnetic parameters.

In the literature there can be found many studies on the effects of deformation conditions, such as a temperature, time, applied force and grain size on the magnetic and mechanical properties of the produced magnets [1-5]. While, there is not much studies in which the subject of discussion are reversal magnetization mechanisms occurring in hot deformed magnets $[6,7]$ and the influence of various production conditions on the $K_{1}$ and $K_{2}$ anisotropy constants.

\footnotetext{
* corresponding author; e-mail: mdospial@wp.pl
}

The aim of this study is to determine the $K_{1}$ and $K_{2}$ magnetocrystalline anisotropy constants and investigations of magnetization reversal mechanisms occurring in hot deformed magnet obtained from $\mathrm{Nd}_{16} \mathrm{Fe}_{78} \mathrm{~B}_{6}$ hard magnetic powder.

\section{Materials and experimental procedure}

In this study, the anisotropic magnets produced from Magnequench MQP-A powder were investigated. The powder was obtained by milling tapes of $\mathrm{Nd}_{16} \mathrm{Fe}_{78} \mathrm{~B}_{6}$ alloy, which were produced using melt-spinning method. The powder was subjected to fractionation using sieves. From the powder with a particle size of $58 \mu \mathrm{m}$, using the hot-pressing method (at $650^{\circ} \mathrm{C}$ ), the isotropic magnets were prepared. The obtained magnets were subsequently subjected to hot deformation $(\varepsilon=65 \%)$, with maintaining of several conditions: applied temperature change, was equal to $500 \frac{{ }^{\circ} \mathrm{C}}{\min }$ and pressure $1.5 \frac{\mathrm{kN}}{\mathrm{s}}$. Both stages of production process (i.e. $M Q 2$ and $M Q 3$ ) were conducted in a vacuum chamber, with pressure of about $10^{-5} \mathrm{~Pa}$. Samples were prepared in Electrotechnical Institute in Wrocław.

Measurements of magnetic hysteresis loops and demagnetization curves in the directions perpendicular and parallel to the magnetization easy axis of the sample were performed using a superconducting vibrating sam- 
ple magnetometer (VSM), in a field of $5 \mathrm{~T}$ (at low frequency vibration of $3.6 \mathrm{~Hz}$ ) with a special noise reducing system [8]. These studies were conducted at the International Laboratory of High Magnetic Fields and Low Temperatures, PAN, in Wrocław.

Experimental dependence, of coercivity from the angle, formed between the magnetization easy axis of the sample and the direction of the external demagnetization field was determined using the LakeShore VSM in a magnetic field up to $2 \mathrm{~T}$. The recoil loops were measured in the demagnetization direction, for parallel and perpendicular angles, to the magnetization easy axis of the sample. Both measurements were performed at the Institute of Physics of Technical University of Częstochowa.

\section{Results and discusion}

Figure 1 shows the magnetic hysteresis loop that was used to estimate the magnetic parameters i.e. coercivity ${ }_{J} H_{\mathrm{c}}=1.21 \mathrm{~T}$, saturation of the polarization $J_{\mathrm{R}}=1.32 \mathrm{~T}$ and maximum magnetic energy product $(B H)_{\max }=$ $360 \frac{\mathrm{kJ}}{\mathrm{m}^{3}}$.

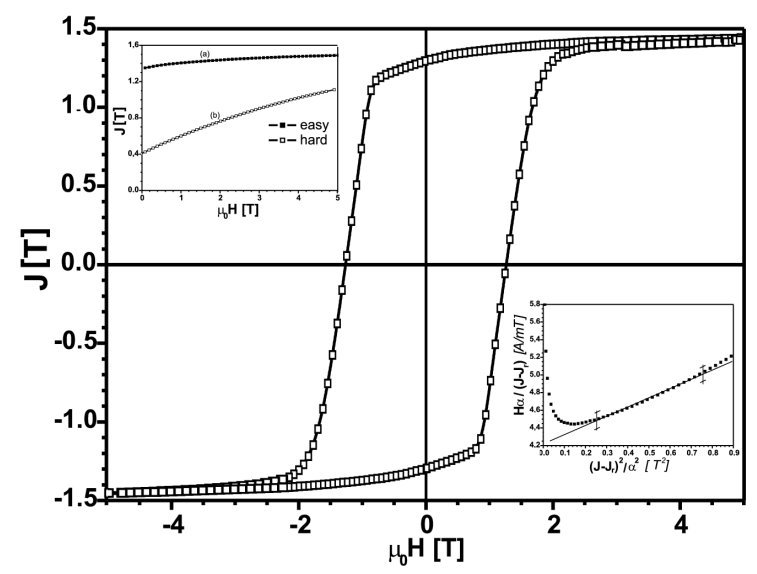

Fig. 1. The magnetic hysteresis loop measured in the direction parallel to easy magnetization axis of the sample; demagnetization curves in the first quadrant of coordinate, measured for demagnetization field applied in the easy and hard magnetization directions (upper inset); linear fit of the Sucksmith-Thompson relationship, modified by Ram and Grunt (lower inset).

In order to determine the saturation of the polarization $J_{\mathrm{S}}=1.41 \mathrm{~T}$ for infinite field, the dependence of polarization $J\left(1 /\left(\mu_{0} H\right)^{2}\right)$, determined from the demagnetization curve in the first quadrant of coordinate axes, for the external magnetic field, applied in direction parallel to easy magnetization direction of the magnet, was used (after extrapolation to infinite field). The anisotropy constants $K_{1}=4.77 \times 10^{6} \frac{\mathrm{J}}{\mathrm{m}^{3}}$ and $K_{2}=1.38 \times 10^{6} \frac{\mathrm{J}}{\mathrm{m}^{3}}$ were determined on the basis of Sucksmith-Thompson relationship, modified by Ram and Grunt [9, 10]:

$$
\frac{H \alpha}{J-J_{r}}=\frac{2 K_{1}}{J_{\mathrm{S}}^{2}}+\frac{4 K_{2}}{J_{\mathrm{S}}^{4}}\left(\frac{J-J_{r}}{\alpha}\right)^{2} .
$$

where $J_{r}$ is polarization in the difficult magnetization direction, $\alpha=\left(J_{\mathrm{S}}-J_{r}\right) / J_{\mathrm{S}}$. The anisotropy constants were evaluated using linear fit, from relation $H \alpha /\left(J-J_{r}\right)$ in function of $\left(J-J_{r}\right) / \alpha^{2}$, which is linear in a certain range of applied magnetic field (Fig. 1, lower inset).

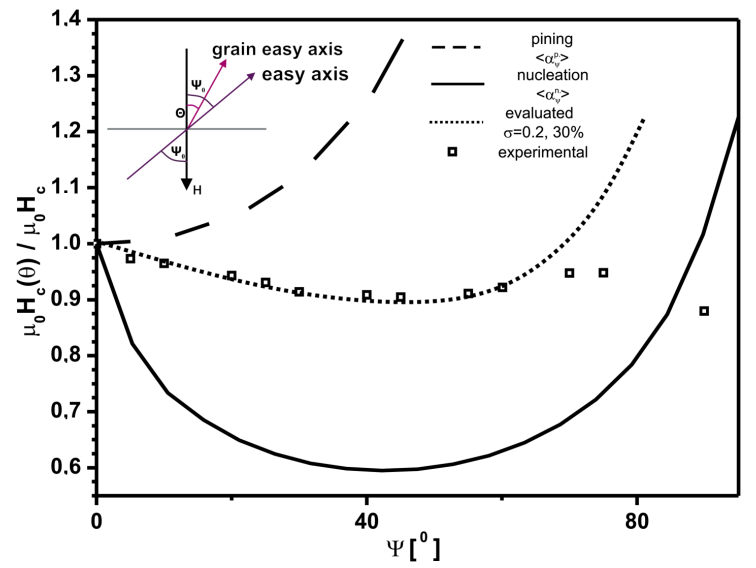

Fig. 2. The dependence of the reduced coercivity of the sample, on the angle between the direction of $\Psi_{0}$ magnetic field and the easy magnetization axis of the sample.

The anisotropy constants were used to determine coercivity dependence as a function of the angle between the easy magnetization axis and the direction of applied magnetic field. Figure 2 presents the comparison of theoretical fit with the experimental results for the dependence of coercivity as a function of the angle between the easy magnetization axis and the direction of applied magnetic field.

From the study carried out i.e. by Durst et al. [11], it can be concluded that in the magnets $\mathrm{Nd}-\mathrm{Fe}-\mathrm{B}$, the probability of finding grains forming the pole angle $\theta$ with the axis of the sample does not depend on the azimuthal angle $\varphi$. What is more, distribution function of grain orientation can be described by Gaussian function. When the direction of the magnetic field is not parallel to the easy magnetization axis of the sample, but forms with it $\Psi_{0}$ angle, then distribution function of grains orientation is expressed by the formula

$$
p\left(\phi, \Psi_{0}\right)=N \exp \left(-\frac{\phi-\Psi_{0}^{2}}{2 \sigma^{2}}\right) .
$$

$N$ is calculated from the normalization condition

$$
N \int_{0}^{\frac{\pi}{2}} p\left(\phi, \Psi_{0}\right) \sin \phi \mathrm{d} \phi=1 .
$$

The average microstructure ratio for the pinning model $\left\langle\alpha_{\Psi}^{\mathrm{p}}\right\rangle$ is calculated from the formula 


$$
\left\langle\alpha_{\Psi}^{\mathrm{p}}\right\rangle=N \int_{0}^{\Psi_{0}} \frac{1}{\cos \phi} \exp \left(-\frac{\left(\Psi_{0}-\phi\right)}{2 \sigma^{2}}\right)^{2} \sin \phi \mathrm{d} \phi
$$

and for the nucleation model $\left\langle\alpha_{\Psi}^{N}\right\rangle$ is calculated from

$$
\begin{aligned}
& \left\langle\alpha_{\Psi}^{n}\right\rangle=N \int_{0}^{\Psi_{0}}\left\{\frac{1}{\cos \theta\left[1+(\tan \theta)^{\frac{2}{3}}\right]^{\frac{3}{2}}}\right. \\
& \left.\times\left[1+\frac{2 K_{2}(\tan \theta)^{\frac{2}{3}}}{K_{1}\left(1+(\tan \theta)^{\frac{2}{3}}\right)}\right]\right\} \\
& \quad \times \mathrm{e}^{\frac{\left.-\Psi_{0}-\theta\right)^{2}}{2 \sigma^{2}}} \sin \theta \mathrm{d} \theta .
\end{aligned}
$$

For large angles $\Psi_{0}$, in the magnetization reversal process there starts to dominate reversible rotation of magnetization vector. For this reason, the coercivity value calculated as a field of opposite polarity, for which the magnetization of the magnet is zero, do not adequately reflect the magnetization reversal process. For the investigated sample, exists good compatibility with the theory if we assume that in $70 \%$ of the grains the main mechanism of coercivity is pinning of domain walls, and in $30 \%$ nucleation of reversed domains. Designated by Lipiec and Davies [1], average grain size for this magnet had bimodal character (small grains up to $250 \mathrm{~nm}$ and large with size of several $\mu \mathrm{m}$ ), which means that a large number of grains is greater than single domain particles. It is therefore possible that they are reversally magnetized by nucleation of reversed domains. Important role in pin- ning of domain walls, therefore is played by structural defects. Best fit of experimental results was obtained for the magnet containing $30 \%$ of the grains with domain structure.

\section{References}

[1] W. Lipiec, H.A. Davies, J. Alloys Comp. 491, 694 (2010).

[2] Y.B. Kim, H.T. Kim, S.H. Cho, G.A. Kapustin, Mater. Sci. 21, 141 (2003).

[3] R.W. Lee, Appl. Phys. Lett. 46, 790 (1985).

[4] C.D. Fuerst, E.G. Brewer, J. Appl. Phys. 73, 5751 (1993).

[5] C.D. Fuerst, E.G. Brewer, J. Appl. Phys. 70, 6444 (1991).

[6] D. Derewnicka-Krawczynska, Ph.D. thesis, Warsaw 2006 (in Polish).

[7] D. Plusa, M. Dospial, D. Derewnicka-Krawczyńska, P. Wieczorek, U. Kolarczyk, Arch. Met. Mater. 56, 159 (2011).

[8] V.I. Nizhankovskii, L.B. Lugansky, Meas. Sci. Technol. 18, 1533 (2007).

[9] W. Sucksmith, J.E. Thompson, Proc. R. Soc. Lond. Ser. A 225, 362 (1954).

[10] U. Ram, P. Grunt, J. Appl. Phys. 54, 2872 (1983).

[11] K.-D. Durst, H. Kronmüller, W. Ervens, Phys. Status Solidi A 108, 403 (1988). 